\title{
Short-term change and prediction of suicidal ideation among adolescents: a daily diary study following psychiatric hospitalization
}

\author{
Ewa K. Czyz, ${ }^{1}$ Adam G. Horwitz, ${ }^{2}$ Alejandra Arango, ${ }^{1,3}$ and Cheryl A King ${ }^{1,3}$ \\ ${ }^{1}$ Department of Psychiatry, University of Michigan, Ann Arbor, MI, USA; ${ }^{2}$ Department of Psychiatry, Rush University \\ Medical Center, Chicago, IL, USA; ${ }^{3}$ Department of Psychology, University of Michigan, Ann Arbor, MI, USA
}

\begin{abstract}
Background: Our understanding of suicidal ideation (SI) and its risk precursors is largely informed by studies spanning over wide time intervals (weeks, months, years). Little is known about SI as it occurs in daily lives of individuals at risk for suicide, the extent to which suicidal thoughts are dynamic over short periods of time, and the degree to which theoretically informed risk factors predict near-term SI. Methods: Thirty-four adolescents hospitalized due to last-month suicide attempt and/or last-week SI (76\% female; ages 13-17) responded to daily surveys sent to their cell phones for four consecutive weeks after discharge ( $n=652$ observations). Results: There was notable variability in day-to-day SI, with half of ideation ratings changing at least one within-person standard deviation from one day to the next. Results of mixed effects models revealed concurrent (same-day), but not shortterm prospective (next-day), associations between SI (frequency, duration, urge) and well-established predictors (connectedness, burdensomeness, hopelessness). However, synergistic effects of low connectedness with either high burdensomeness or high hopelessness were reliably associated with more severe same- and next-day suicidal ideation. Conclusions: This study adds to emerging literature indicating that suicidal thoughts fluctuate considerably among individuals at risk for suicide, further extending it by focusing on adolescents in the critical posthospitalization period. Fostering high-risk adolescents' sense of connectedness to others may be an especially promising intervention target. Frequent assessment of SI and its predictors, independently and in combination, could help identify promising predictors of short-term risk and meaningful intervention targets in high-risk teens. Keywords: Ecological momentary assessment; adolescents; suicidal ideation; hopelessness; interpersonal-
\end{abstract} psychological theory.

\section{Introduction}

Suicide is currently the second leading cause of death among adolescents in the United States (Centers for Disease Control and Prevention, 2018). Moreover, approximately $17 \%$ of adolescents surveyed nationally reported thoughts of suicide in the past year and $7.4 \%$ attempted suicide (Kann et al., 2018). Research spanning several decades has provided valuable information about risk and protective factors associated with suicidal thoughts and behavior (Bridge, Goldstein, \& Brent, 2006; McLoughlin, Gould, \& Malone, 2015; Spirito \& EspositoSmythers, 2006). At the same time, there remain unanswered questions about the near-term precursors of suicidal ideation (SI) and behavior. On an even more fundamental level, little is known about the prevalence of these phenomena in individuals' daily lives (Nock, Prinstein, \& Sterba, 2009).

The need to fill these knowledge gaps is especially critical for psychiatrically hospitalized teens, as the postdischarge period is often characterized by ongoing suicide risk-related crises (Brent et al., 2013; Czyz, Berona, \& King, 2016; Goldston et al., 1999; Yen et al., 2013) and fluctuating risk patterns (Czyz \& King, 2015; Prinstein et al., 2008). Recently discharged individuals are also at an increased risk for

Conflict of interest statement: No conflicts declared. suicide (Chung et al., 2017). Given that the majority of existing studies focusing on SI and suicide attempts have relied on longer assessment windows (weeks, months, years), with the average follow-up period being nearly 7 years (Ribeiro et al., 2016), there is limited information about the extent to which theoretically- and clinically- informed risk indicators are meaningfully associated with SI or behavior in the near term. In other words, relying on longer time intervals between assessments has precluded gaining clinically relevant insight about who might be at imminent or acute suicide risk and when (Bagge, Glenn, \& Lee, 2013; Glenn \& Nock, 2014; Rudd et al., 2006).

\section{SI in individuals' daily life}

Recent advances in technology, particularly with respect to mobile phones, have made it increasingly possible to investigate the occurrence and precursors of SI and behavior in real-time via Ecological Momentary Assessment (EMA), which involves repeated and frequent assessment of behavior in the person's natural environment (Moskowitz \& Young, 2006; Shiffman, Stone, \& Hufford, 2008). Existing studies involving repeated assessment of suicide risk-related constructs have demonstrated that EMA data collection is feasible (Davidson, Anestis, \& Gutierrez, 2017) and that repeated assessment of suicidal thoughts 
was not associated with any iatrogenic increase in suicidal thinking (Husky et al., 2014; Law et al., 2015). Additional EMA studies with adults have indicated that suicidal thoughts are dynamic over short periods of time, sometimes fluctuating over an interval of only a few hours (Hallensleben et al., 2018; Kleiman et al., 2017).

In addition to improving our understanding of fluctuations in SI, the use of EMA approaches has allowed for a more nuanced assessment of proximal risk factors for suicidal thoughts and behaviors. A study by Husky et al. (2017) identified contextual variables associated with SI among recently discharged adult patients and found that doing work, passive leisure, and inactivity were associated with increased risk for SI. Being alone also increased risk, whereas being with close others reduced this risk. Ben-Zeev, Young, \& Depp (2012) assessed depressed adult inpatients multiple times per day and found that momentary ratings of sadness, tension, and boredom predicted SI at the next assessment point, above and beyond the influence of SI at the previous assessment. In contrast, known risk factors such as hopelessness, guilt, and anhedonia did not predict short-term SI. Similarly, in two adult samples of previous suicide attempters and psychiatric inpatients, Kleiman et al. (2017) found that while hopelessness, burdensomeness, and loneliness had strong associations with concurrent SI, these risk factors were not associated with next-assessment SI when controlling for SI at the previous assessment. Although these studies have contributed to what is known about SI in the nearterm, little is known about short-term changes in SI, as well as predictors of these changes, in recently discharged adolescents. To our knowledge, only one EMA study (Nock et al., 2009) has assessed SI in adolescents (ages 12-19), however, participants were recruited from the community and reported relatively infrequent SI over the 2-week follow-up period.

\section{Purpose of this study}

In our previous study of adolescents who were followed daily for a month following psychiatric hospitalization, we learned that daily assessments of SI in the critical postdischarge period were feasible, acceptable to adolescents, and resulted in more teens reporting thoughts of suicide compared to an end-ofstudy assessment (Czyz, King, \& Nahum-Shani, 2018). Encouraged by these findings-and relying on the same sample of adolescents, which was originally drawn from a pilot trial of a brief intervention for hospitalized teens (Czyz, King, \& Biermann, 2018)the current study provides a nuanced examination of the postdischarge experience among suicidal adolescents. Specifically, we sought to investigate, in a more fine-grained manner, the extent to which SI indicators (i.e. frequency, duration, and urge severity) and theoretically informed suicide risk and protective factors (i.e. connectedness, burdensomeness, hopelessness) are dynamic or vary day-to-day in the postdischarge period. In addition, we examined the degree to which hopelessness, connectedness, and burdensomeness are associated, independently and in combination, with same-day and next-day SI frequency, duration, and urge severity. These questions build on existing EMA research with adults at risk for suicide (Ben-Zeev et al., 2012; Hallensleben et al., 2018; Husky et al., 2017; Kleiman et al., 2017) and expand on it by explicitly focusing on the postdischarge period among high-risk adolescents.

Our focus on connectedness and burdensomeness is informed by the interpersonal-psychological theory of suicidal behavior (IPTS) (Joiner, 2005; Van Orden et al., 2010), wherein suicidal desire is conceptualized as arising from low levels of social connectedness (i.e., thwarted belongingness) and perception that one is a burden on others (burdensomeness). IPTS proposes that the combination of these two factors leads to suicidal desire, which has been supported by previous research among adults and teens (Chu et al., 2017; Opperman, Czyz, Gipson, \& King, 2015; Stewart, Eaddy, Horton, Hughes, \& Kennard, 2017). We thus expected that the interaction between low connectedness and high burdensomeness to be associated with greater severity of SI. Given that the combination of thwarted belongingness and burdensomeness has been proposed to serve as proximal pathway from a number of different risk factors to SI (Van Orden et al., 2010), evaluating same- and nextday relationship between the simultaneous presence of these constructs and SI is especially fitting.

Our focus on hopelessness, defined as negative expectations about the future, is guided by a cognitive framework (Wenzel \& Beck, 2008) wherein hopelessness is conceptualized as both central and proximal to the emergence of SI. Moreover, the link between hopelessness and SI and behavior is also well-documented in previous work (e.g., Beck, Steer, Kovacs, \& Garrison, 1985; Mazza \& Reynolds, 1998; Smith, Alloy, \& Abramson, 2006; Steer, Kumar, \& Beck, 1993). Consistent with theory and previous work, we expected that hopelessness to be associated with greater severity of SI.

This study also builds and expands on Kleiman et al.'s study (2017) by considering both the main effects of burdensomeness, connectedness, and hopelessness as well as their two-way interaction in the short-term prediction of SI. Specifically, to consider the possibility that concurrent or short-term prospective prediction is improved when the predictors of burdensomeness, connectedness, and hopelessness are considered in any combination -rather than the specific combination of low connectedness and burdensomeness, as proposed by IPTS-we explored all possible two-way interactions between these constructs. The importance of considering synergistic effects is also consistent with theoretical models of suicidal behavior and recent meta-analytic studies indicating that known individual risk factors 
appear to have a modest relationship with suicidal thoughts and behavior (Franklin et al., 2017; Ribeiro et al., 2016). Finally, examining these relationships concurrently (same-day) and prospectively (next-day) will allow for a greater understanding of the dynamic, or more lasting, relationship between different suicide risk indicators and suicidal thoughts. While previous research has provided initial evidence that certain factors appear to have a time-dependent relationship with SI (Ben-Zeev et al., 2012; Kleiman et al., 2017), investigating the extent to which this holds across other at-risk populations is warranted.

\section{Methods}

\section{Participants}

Participants included adolescents (ages 13-17) who were psychiatrically hospitalized due to last-month suicide attempt and/or last-week SI. Participants were recruited between January and May 2017 to take part in a pilot study of a brief psychosocial intervention-focused on feasibility and acceptability of a three-component (individual and family sessions, postdischarge call) motivational interviewing (MI)-enhanced safety planning intervention compared to treatment as usual that included safety planning without MI - with a daily survey follow-up component (Czyz, King, \& Biermann, 2018). Exclusion criteria included: severe cognitive impairment or altered mental status (e.g., active psychosis or mania), transfer to medical unit or residential placement, no availability of a legal guardian (ward of state), and teen not having a cell phone with text messaging capability. Of the 50 potentially eligible participants, based on an initial screening of admission records and consultation with inpatient team as needed, two (4\%) did not own a cell phone and one (2\%) did not have cell phone access for disciplinary reasons. Of those meeting all eligibility criteria, $36(76.6 \%)$ provided parental consent and teen assent. This study's analytic sample includes 34 adolescents who continued in the study (one teen withdrew from the study and one teen no longer had a cell phone).

\section{Procedures}

The study was approved by the participating university's Institutional Review Board. After providing consent and assent, participants completed a series of self-report surveys during hospitalization in addition to three follow-up assessments: approximately 1-2 weeks, 1 month, and 3 months after hospitalization. In addition, beginning on the first day after the discharge, participating adolescents were also asked to complete one survey each evening for 28 consecutive days. A link to the survey, developed using a Qualtrics survey tool (www.qualtrics.c om), was sent to participants' phones via text messages between $5 \mathrm{pm}$ and $7 \mathrm{pm}$. Participants were asked to respond to the survey within $1-1.5 \mathrm{hr}$. Text messages were sent automatically using a secure research platform called TelEMA (Fernandez, Johnson, \& Rodebaugh, 2013). Participants had the option to fill out the survey on their smartphone or using an internet browser on a computer, allowing for the survey to be mobile compatible while ensuring that participants could respond even if internet access on their smartphone was restricted or if they did not own a smartphone. Responses to daily surveys were monitored by the study's on-call clinicians who contacted participants if they endorsed current ideation with intent/plan or a suicide attempt in last $24 \mathrm{hr}$; for additional risk management details, please see Czyz, King, \& Nahum-Shani, 2018. Participants were compensated $\$ 20$ to offset text messaging and/or data plan fees in addition to $\$ 4$ for each completed survey. With the exception of pertinent baseline information, this study's focus is on the data obtained via daily surveys.

\section{Measures}

Hopelessness. In reference to the last $24 \mathrm{hr}$, adolescents rated the extent to which they felt hopeless ('I see only bad things ahead of me, not good things') using a 4-point scale ranging from 'strongly disagree' to 'strongly agree.' This item was from the 6item Brief Hopelessness Scale (Bolland, McCallum, Lian, Bailey, \& Rowan, 2001), adapted from the Hopelessness Scale for Children (Kazdin, Rodgers, \& Colbus, 1986). The measure has sound psychometrics and is comparable to the full measure of hopelessness. This item was selected because it had strong factor loading as well as item-total scale correlation (Kazdin et al., 1986).

Connectedness and burdensomeness. Referencing the last 24-hr, adolescents were asked each day to rate, using a 7 -point scale (from 'not at all true for me' to 'very true for me'), the extent of their sense of connectedness to others ('I am close to other people') and the extent of their sense of burdensomeness ('The people in my life would be happier without me'). These two questions were derived from the 15-item Interpersonal Needs Questionnaire (INQ) (Van Orden, Cukrowicz, Witte, \& Joiner, 2012) measuring thwarted belongingness and perceived burdensomeness. The INQ has strong psychometric properties in adult and adolescent samples (Hill et al., 2015; Van Orden et al., 2012). For the purposes of creating a brief assessment of daily connectedness and burdensomeness, we chose these items due to their strong factor loading on their respective subscales among young adults (Van Orden et al., 2012) and adolescents (January 2016, Hill personal communication).

Suicidal ideation. Baseline SI severity, based on the Columbia-Suicide Severity Rating Scale (C-SSRS) (Posner et al., 2011), was assessed on a 6-point scale (from 'wish to be dead' to 'SI with specific plan and intent'). The C-SSRS assesses a range of suicidal thoughts and behaviors, and it has shown strong reliability and validity (Posner et al., 2011), including predictive validity for suicide attempts (Gipson, Agarwala, Opperman, Horwitz, \& King, 2015; Horwitz, Czyz, \& King, 2015).

Each day, adolescents were asked about SI: 'At any point in the last $24 \mathrm{hr}$, did you have any thoughts of killing yourself?' An affirmative answer was followed by questions modeled after the C-SSRS (Posner et al., 2011) assessing frequency ('How many times did you have thoughts of killing yourself?') and duration ('How long did these thoughts last?'). Using a 4-point scale, frequency response options ranged from 'only one time' to 'all the time.' Using a 5 point-scale, duration response options ranged from 'a few seconds or minutes' to 'more than $8 \mathrm{hr} /$ continuous.' In addition, we asked participants to rate, using a scale of 1 (low) to 7 (high), how intense their urge to act on suicidal thoughts was ('How strong was the urge to act on your thoughts of suicide?'). The phrasing of the question was modeled after an item assessing urge to engage in self-injurious behaviors used in another EMA study (Nock et al., 2009). We created three continuous scales assessing different aspects of SI (frequency, duration, and urge severity). It is important to note that teens reporting no presence of SI on a given day responded to an equivalent number of follow-up 'filler' items to avoid inadvertently encouraging teens to underreport SI based on survey length.

\section{Data analysis}

We provide descriptive statistics, including means and standard deviations, for the three SI indicators (frequency, 
duration, urge severity) and their three predictors (connectedness, burdensomeness, hopelessness). To examine the extent to which these variables varied day-to-day across the 28-day follow-up period, we considered interclass correlations (ICC), which provide information about the proportion of variance being due to between-person variability. Within-person variability (I-ICC) was obtained to evaluate the proportion of variance due to day-to-day change in SI or its predictors. We also provide a graphic representation of the extent to which SI and its predictors varied across time by plotting group meancentered (i.e., each participant's own mean is centered at 0) time-series plots, standardizing variability over time for the entire sample. Finally, we describe the extent to which SI ratings, as well as its predictors, changed from one day to the next day by at least one standard deviation from participants' individual group mean (i.e., within-person standard deviation). These approaches to describing and visualizing variability are consistent with Kleiman et al. (2017).

In order to explore the relationship between connectedness, burdensomeness, and hopelessness-as well as their two-way interactions-with same-day and next-day SI frequency, duration, and urge severity, we utilized linear mixed effects models. All models included the three predictors, and next-day models also controlled for previous-day SI corresponding to the outcome (e.g., for next-day duration, previous-day duration was controlled for). All models included a random intercept and slope and, because the data came from a pilot intervention study, also controlled for any group effects $^{2}$. To adjust for multiple comparisons, we applied the Benjamini-Hochberg procedure (Benjamini \& Hochberg, $1995)$ with a 0.05 false positive discovery rate. Analyses were conducted using SAS (version 9.4) (SAS Institute, Inc.: Cary, NC, USA).

\section{Results}

\section{Participant characteristics}

The sample's sex distribution included $76.5 \%$ $(n=26)$ female adolescents, with a mean age of 15.5 years $(S D=1.35)$. The sample's racial/ethnic distribution was as follows (more than one category could be selected): $85.3 \%(n=29)$ Caucasian, $8.8 \%$ $(n=3)$ African-American/Black, $8.8 \%(n=3)$ Asian, $5.9 \%(n=2)$ Hispanic, $2.9 \%(n=1)$ American Indian or Alaska Native, and $2.9 \%(n=1)$ Native Hawaiian or Other Pacific Islander. At baseline, 52.9\% $(n=18)$ of the teens had a previous suicide attempt; $32.4 \%$ $(n=11)$ attempted within a month of hospitalization. The average baseline SI severity (range 0-5) was 4.06 $(S D=0.92)$. Approximately a third $(32.4 \% ; n=11)$ of the $^{1}$ sample had at least one previous psychiatric hospitalization. Obtained via chart review, participants received the following psychiatric diagnoses: a depressive disorder, including major depressive or unspecified depressive disorder, (85.3\%; $n=29)$; an anxiety disorder, including social anxiety, generalized anxiety, or unspecified anxiety disorder, (70.6\%; $n=24) ;$ attention-deficit hyperactivity disorder $(17.6 \% ; n=6)$; unspecified mood disorder (11.8\%; $n=4)$; posttraumatic stress disorder $(11.8 \% ; n=4)$; obsessive-compulsive disorder $(2.9 \% ; n=1)$; and polysubstance abuse disorder $(2.9 \%, n=1)$. With regard to survey adherence, participants responded to $654(69.4 \%)$ and completed 650 (68.9\%) surveys during the 28-day period. On average, participants completed $19.12(S D=7.07)$ surveys.

\section{Description of SI indicators and their predictors}

Presence of any SI was reported in $24.4 \%$ of all responses ( $n=159$ days or events) over the 28-day follow-up; a total of $24(70.6 \%)$ individual participants endorsed ideation at least once across these 159 events. The proportion of disclosures of any SI was fairly consistent across the four weeks: $24.6 \%$ in week 1 ( $n=46$ events), 22.1\% ( $n=38$ events $)$ in week 2, 24\% ( $n=36$ events) in week 3 , and $27.3 \%$ ( $n=39$ events) in week 4 . The means, standard deviations, and ICCs for SI frequency, duration, urge and predictor variables (connectedness, burdensomeness, hopelessness) are presented in Table 1. The ICCs presented in Table 1 indicate that the three SI indicators as well as their predictors varied considerably from day to day. Specifically, 43\%, $46 \%$, and $47 \%$ of the variance in ideation frequency, duration, and urge, respectively, was due to withinperson (1-ICC) variability. The within-person or dayto-day variability for connectedness, burdensomeness, and hopelessness ranged, respectively, from $37 \%$ to $31 \%$ to $33 \%$. Figures 1 and 2 show graphically the day-to-day variability in SI indicators as well as their predictors. The scores are group-mean centered (scores are subtracted from each participants' overall or group mean), which allows for depicting the variability around a standardized mean for all participants.

Participants' SI varied from one day to the next. Specifically, for SI frequency, duration, and urge, $54.7 \% \quad(n=235 / 519), \quad 44 \% \quad(n=229 / 520)$, and $43.3 \% \quad(n=225 / 520)$ of daily ideation ratings, respectively, changed from one day to the next day by at least one standard deviation from participants' individual mean. This suggest that participants' dayto-day level of ideation differed considerably nearly half the time, with all participants having at least one instance of SI change by at least one within-person standard deviation from one day to the next day.

Participants' levels of connectedness, burdensomeness, and hopelessness also changed from one day to the next consecutive day, however less so than for SI. Approximately a third of connectedness, burdensomeness, and hopelessness ratings differed

Table 1 Descriptive statistics for suicidal ideation indicators and their predictors

\begin{tabular}{lccc}
\hline Variables (range) & Mean & SD & ICC \\
\hline Connectedness (1-7) & 5.17 & 1.49 & .63 \\
Burdensomeness (1-7) & 3.03 & 1.79 & .69 \\
Hopelessness (1-4) & 2.13 & 0.90 & .67 \\
Suicidal ideation (SI) frequency (0-4) & 0.68 & 1.30 & .57 \\
SI duration (0-5) & 0.76 & 1.51 & .54 \\
SI urge severity (0-7) & 1.03 & 2.06 & .53 \\
\hline
\end{tabular}

ICC, interclass correlation. 


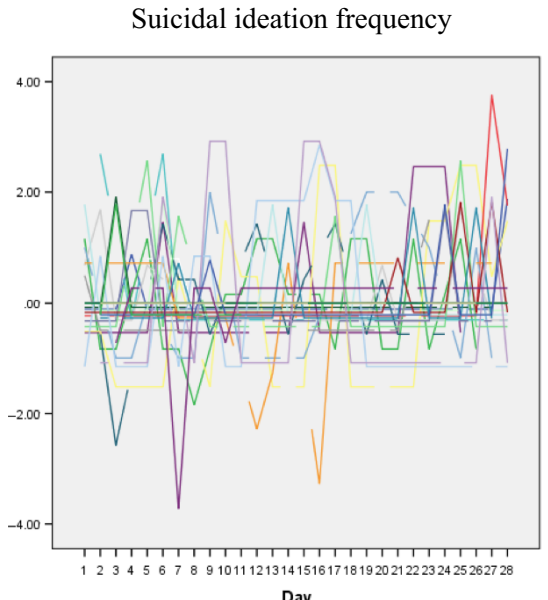

Day

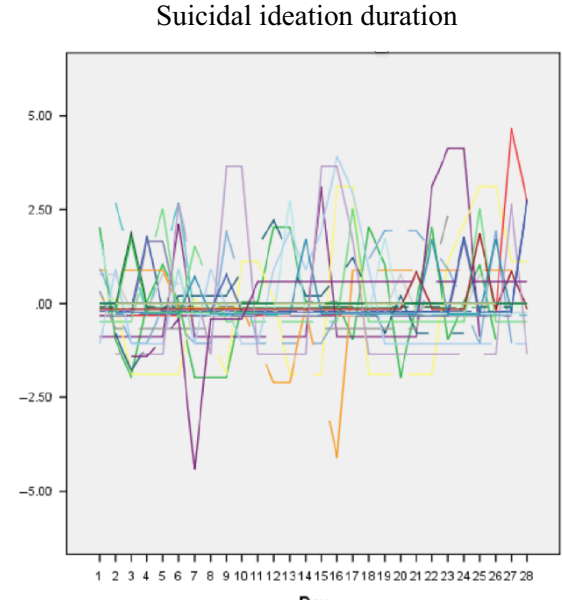

Day
Suicidal ideation urge severity

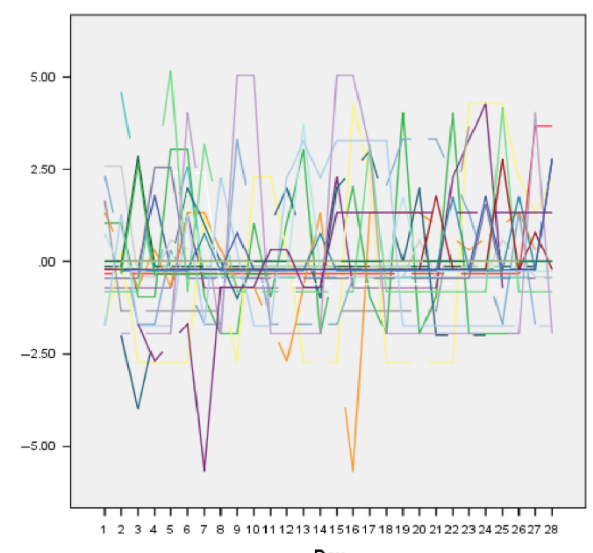

Day

Figure 1 Ideation time-series plots. Note: Variables are group-mean centered [Colour figure can be viewed at wileyonlinelibrary.com]
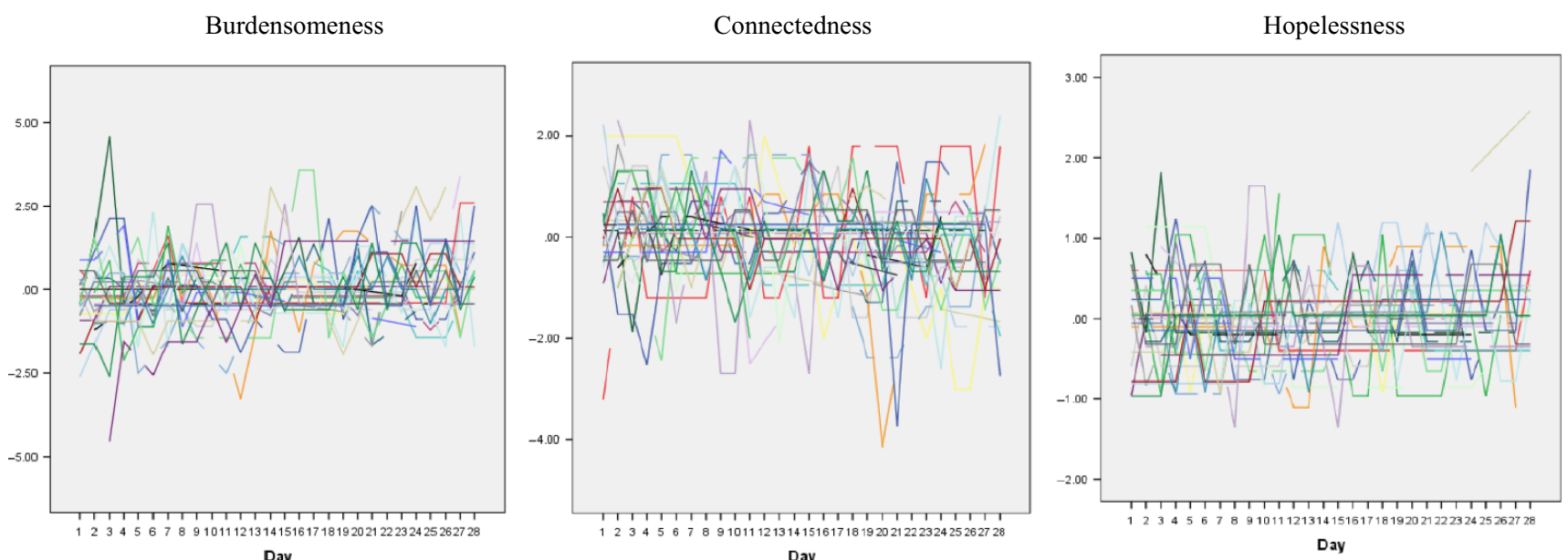

Figure 2 Predictors time-series plots. Note: Variables are group-mean centered [Colour figure can be viewed at wileyonlinelibrary.com]

from one day to the next by at least one withinperson standard deviation: 33.9\% (173/510) for connectedness, $34.5 \%(176 / 510)$ for burdensomeness, and $32.4 \%(166 / 512)$ for hopelessness. Nearly all participants had at least one instance of dayto-day change in these variables $1100 \%$ for connectedness; $94.1 \%$ for burdensomeness; $97.1 \%$ for hopelessness) by at least one within-person standard deviation.

\section{Prediction of same-day and next-day SI}

Results from mixed effects models (Table 2) indicate statistically significant concurrent, but not shortterm prospective, relationships between SI outcomes and lower connectedness, greater burdensomeness, and higher hopelessness. Only previous-day SI was independently associated with next-day ideation indicators.

Also summarized in Table 2 are results from mixed effects models considering two-way interactions between the three predictors and SI. When considered concurrently, all three two-way interactions were significantly associated with SI frequency, duration, and urge severity. However, when examined prospectively and adjusting for previous day SI, only the combination of connectedness with burdensomeness was significantly associated with next-day SI frequency, duration, and urge severity. In addition, the interaction between connectedness and hopelessness was significantly associated with next-day SI frequency and duration. As shown in Figure 3, when either burdensomeness or hopelessness were high, presence of higher levels of connectedness was associated with decreasing same-day SI; the same pattern emerged when interactions were plotted for nextday SI. Finally, as shown in Figure 3, the combination of high burdensomeness and high hopelessness was associated with increasing same-day SI frequency, duration, and urge severity.

\section{Discussion}

In this study of psychiatrically hospitalized teens followed daily for a month after discharge, we examined 
Table 2 Mixed effects models examining independent and combined predictors of same-day and next-day SI

\begin{tabular}{|c|c|c|c|c|c|c|c|c|}
\hline & \multicolumn{4}{|c|}{ Same-day SI frequency $(N=651)$} & \multicolumn{4}{|c|}{ Next-day SI frequency $(N=509)$} \\
\hline & $\mathrm{B}$ & $S E$ & Stand. B & $p$ & $\mathrm{~B}$ & $S E$ & Stand. $B$ & $p$ \\
\hline Connectedness & -0.16 & 0.04 & -0.19 & $<.001$ & -0.06 & 0.05 & -0.07 & .174 \\
\hline Burdensomeness & 0.11 & 0.04 & 0.15 & .003 & 0.06 & 0.04 & 0.08 & .158 \\
\hline Hopelessness & 0.37 & 0.07 & 0.26 & $<.001$ & 0.01 & 0.08 & 0.01 & .870 \\
\hline SI & & & & & 0.24 & .05 & 0.24 & $<.001$ \\
\hline \multicolumn{9}{|l|}{ Interaction models ${ }^{a}$} \\
\hline Connectedness $\times$ Burdensomeness & -0.06 & 0.01 & -0.13 & $<.001$ & -0.06 & 0.02 & -0.11 & .001 \\
\hline Connectedness $\times$ Hopelessness & -0.09 & 0.03 & -0.09 & .002 & -0.08 & 0.03 & -0.08 & .016 \\
\hline \multirow[t]{3}{*}{ Burdensomeness $\times$ Hopelessness } & 0.08 & 0.03 & 0.10 & .002 & -0.01 & 0.03 & -0.01 & .849 \\
\hline & \multicolumn{4}{|c|}{ Same-day SI duration $(N=652)$} & \multicolumn{4}{|c|}{ Next-day SI duration $(N=509)$} \\
\hline & $\mathrm{B}$ & $\mathrm{SE}$ & Stand. B & $p$ & $\mathrm{~B}$ & $\mathrm{SE}$ & Stand. B & $p$ \\
\hline \multicolumn{9}{|l|}{ Main effects model } \\
\hline Connectedness & -0.18 & 0.04 & -0.18 & $<.001$ & -0.07 & .05 & -0.07 & .162 \\
\hline Burdensomeness & 0.16 & 0.04 & 0.19 & $<.001$ & 0.10 & 0.05 & 0.12 & .038 \\
\hline Hopelessness & 0.37 & 0.08 & 0.22 & $<.001$ & -0.01 & 0.09 & -0.01 & .912 \\
\hline SI & & & & & 0.29 & 0.05 & 0.29 & $<.001$ \\
\hline \multicolumn{9}{|l|}{ Interaction models ${ }^{\mathrm{a}}$} \\
\hline Connectedness $\times$ Burdensomeness & -0.08 & 0.02 & -0.15 & $<.001$ & -0.06 & 0.02 & -0.11 & .001 \\
\hline Connectedness $\times$ Hopelessness & -0.10 & 0.03 & -0.09 & .002 & -0.09 & 0.04 & -0.08 & .018 \\
\hline \multirow[t]{3}{*}{ Burdensomeness $\times$ Hopelessness } & 0.08 & 0.03 & 0.09 & .005 & 0.001 & 0.03 & 0.001 & .974 \\
\hline & \multicolumn{4}{|c|}{ Same-day SI urge $(N=652)$} & \multicolumn{4}{|c|}{ Next-day SI urge $(N=509)$} \\
\hline & B & $\mathrm{SE}$ & Stand. B & $p$ & $\mathrm{~B}$ & $\mathrm{SE}$ & Stand. B & $p$ \\
\hline \multicolumn{9}{|l|}{ Main effects model } \\
\hline Connectedness & -0.27 & 0.06 & -0.20 & $<.001$ & -0.09 & 0.07 & -0.07 & .213 \\
\hline Burdensomeness & 0.25 & 0.06 & 0.21 & $<.001$ & 0.13 & 0.07 & 0.12 & .053 \\
\hline \multicolumn{9}{|l|}{ Interaction models ${ }^{a}$} \\
\hline Connectedness $\times$ Burdensomeness & -0.14 & 0.02 & -0.18 & $<.001$ & -0.10 & 0.03 & -0.13 & $<.001$ \\
\hline Connectedness $\times$ Hopelessness & -0.21 & 0.04 & -0.14 & $<.001$ & -0.10 & 0.05 & -0.07 & .056 \\
\hline Burdensomeness $\times$ Hopelessness & 0.22 & 0.04 & 0.18 & $<.001$ & -0.04 & 0.05 & -0.03 & .459 \\
\hline
\end{tabular}

SI, Suicidal Ideation.

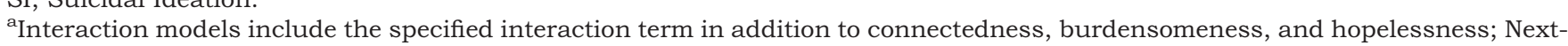
day interaction models additionally control for previous-day SI; All models also control for group; Bolded p values meet statistical significance threshold with adjustment for multiple comparisons.

concurrent and short-term prospective associations between three indicators of SI severity (frequency, duration, and urge) and theoretically-informed risk precursors: connectedness, burdensomeness, and hopelessness. Given limited information about the extent to which SI and its precursors change over short periods of time among adolescents in the postdischarge period, we also examined day-to-day variability in SI as well as connectedness, burdensomeness, and hopelessness. To our knowledge, this is the first study employing fine-grained (i.e., daily) assessments among discharged adolescents to understand the dynamic
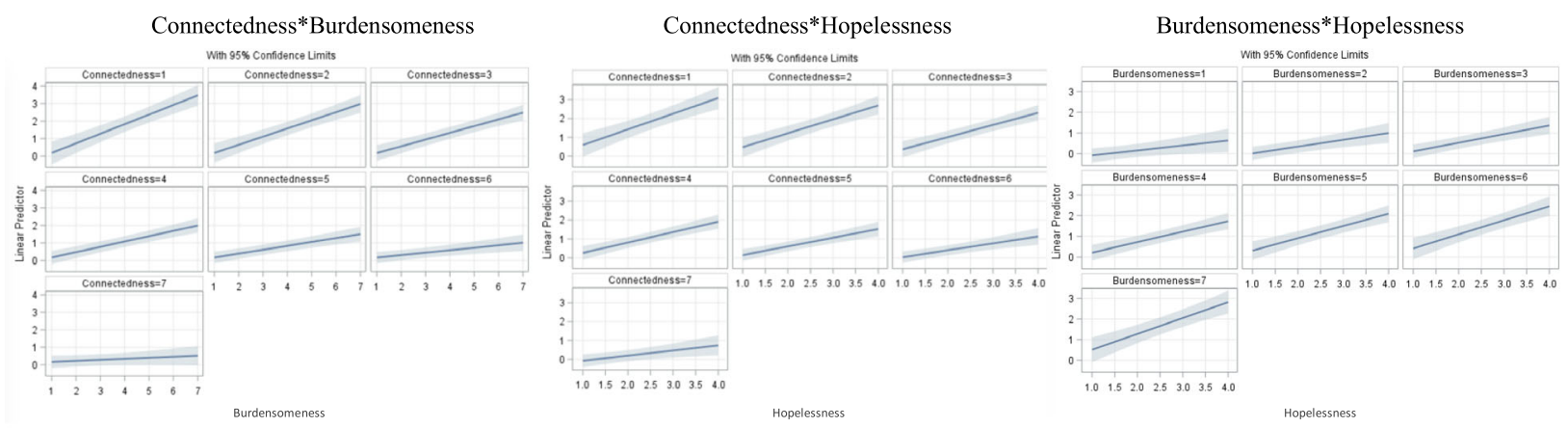

Figure 3 Same-day suicidal ideation interactions. Note: Interactions are for suicidal ideation duration. Corresponding interactions for ideation frequency and urge severity had consistent patterns [Colour figure can be viewed at wileyonlinelibrary.com] 
nature of these phenomena and short-term prediction of SI.

Several key findings emerged from this study. First, day-to-day variability in SI frequency, duration, and urge severity was notable. We found that between $43 \%$ and $47 \%$ of the variance in SI was due to withinperson, or day-to-day, variability. This is consistent with previous work conducted by Kleiman et al. (2017) who, in two samples of adults responding to four surveys per day, reported that $33 \%$ and $47 \%$ of variance was accounted for by within-person variability. Thus, this finding contributes to the growing body of literature suggesting that SI is dynamic over short periods of time. In fact, about half of SI ratings in this study changed at least one within-person standard deviation from one day to the next. In line with IPTS theory, which conceptualizes these as dynamic (Van Orden et al., 2010), it is notable that the precursors to SI examined in this study also varied considerably, with approximately a third of connectedness, burdensomeness, and hopelessness ratings changing day-to-day by at least one within-person standard deviation. This pattern of variability is worth noting because most studies ask about severity of SI as well as risk indicators over longer time intervals that may not capture these changes or states, which, in turn, could be critical to improving risk detection.

Additional findings that warrant discussion include the fact that, despite significant concurrent associations, connectedness, burdensomeness, and hopelessness were not significant predictors of nextday SI indicators when accounting for previous-day SI. These time-dependent relationships may be reflective of the dynamic, or state-like, nature of these constructs and, while contrary with what might be expected based on theory and evidence found in existing literature, it may be that hopelessness, burdensomeness, and connectedness are limited in predicting short-term SI. This is consistent with Ben-Zeev et al. (2012) who found no relationship between hopelessness and near-term ideation as well as with Kleiman et al.'s study (2017) where only SI (but not hopelessness, loneliness, or burdensomeness) predicted short-term changes in SI assessed multiple times per day. In this study, previous-day SI was also associated with next-day SI. This suggests that, despite fluctuating day-today, greater severity of SI is a strong indicator of ideation the following day.

Although not associated independently, our findings indicate that particular combinations of risk indicators predicted next-day suicidal thoughts. Specifically, low connectedness in combination with either high burdensomeness or high hopelessness was significantly associated with next-day SI, even when controlling for previous-day SI. The combination of low connectedness and high burdensomeness predicting ideation is in line with IPTS (Joiner, 2005; Van Orden et al., 2010) as well as a number of studies (Chu et al., 2017; Opperman et al., 2015;
Stewart et al., 2017). However, to our knowledge, this study is the first to provide evidence supporting the importance of these two theory components to our understanding of SI or desire using a short-term prospective design. Moving beyond IPTS, however, it is noteworthy that low connectedness together with high hopelessness was also associated with next-day SI, pointing to multiple proximal pathways to SI. Although a sense of burdensomeness may contribute to hopelessness, hopelessness is a broader construct that may reflect struggles in interpersonal and/or noninterpersonal domains. With implications for our understanding of suicide risk, low connectedness, together with elevated sense of burdensomeness or hopelessness, appears to represent an especially risky state for not only the emergence of SI but also its persistence into the next day. The critical role of connectedness in these synergistic effects is further supported by our finding that the combination of hopelessness and burdensomeness did not predict next-day ideation. Additional studies are needed to determine if this pattern of findings can be replicated in other samples.

The study findings should be considered in light of its limitations. The primarily female and Caucasian sample limits generalizability of the results. Although comparable to EMA studies of similar length (e.g. 62.0\% reported by Kleiman et al., 2017; $77.8 \%$ reported by Torous et al., 2015), survey adherence (69\%) may be another limitation, as it is not possible to rule out that adherence may have been influenced by relevant mood states or factors. Moreover, although single-item measures are commonly used in context of EMA studies to lower response burden, the extent to which these items correspond to longer measures should be examined in future research. Daily surveys employed in this study are subject to greater recall bias relative to multiple daily assessments, which would have the advantage of providing even more fine-grained information about teens' experiences. Our work should be replicated not only across different samples but also in studies utilizing more frequent assessment schedules. This study was also not sufficiently powered to consider between-person moderators of repeatedly assessed experiences, such as sex, gender identify, and clinical characteristics (e.g., attempt history). There is also possible concern that teens underreported thoughts of suicide. Although this possibility exists, we found that significantly more teens in this sample reported thoughts of suicide via daily diaries compared to an end-of-study assessment covering the same time period (Czyz, King, \& Nahum-Shani, 2018), suggesting willingness to disclose this information. Finally, the measure of suicidal thoughts used in this study did not assess suicidal intent or plans, or, more generally, variability in the content of suicidal thoughts, which could be important to our understanding of transitions in suicidal thinking and precursors to suicidal 
behavior. Future work should also examine comprehensive theoretical frameworks concerned with transition in suicidal thinking (e.g., in IPTS, hopelessness about burdensomeness and low connectedness is hypothesized to explain the transition from passive to active ideation).

\section{Conclusions}

Taken together, this study adds to the growing literature suggesting that suicidal thoughts fluctuate considerably, thus highlighting the value of EMA methods in providing a more nuanced understanding of when these thoughts occur as well as the associated contextual factors. The dynamic nature of SI observed in this study also converges with clinical recommendations to closely monitor ideation in the postdischarge period, although much more work is needed to address critical issues related to the feasibility of clinical implementation of more frequent monitoring. Moreover, these findings point to the importance of additional research to determine if interventions targeting dynamically changing levels of SI and its predictors in real time, such as via justin-time adaptive interventions (Nahum-Shani, Hekler, \& Spruijt-Metz, 2015; Nahum-Shani et al., 2017), would prove effective. Interventions targeting burdensomeness, hopelessness, and connectedness might be especially valuable in reducing the intensity of ideation occurring in close proximity, i.e., the same day. Because connectedness moderated, both concurrently and short-term prospectively, the impact of hopelessness and burdensomeness on ideation, fostering high-risk adolescents' sense of connectedness may be an especially promising intervention target. Increasing social support, particularly in the domain of the family, is often a key focus of interventions for adolescents at risk for suicide, including our MI-enhanced safety planning intervention pilot (Czyz, King, \& Biermann, 2018). However, to inform tailored interventions for adolescents at suicide risk and identify the most promising mechanisms of change, it would be informative to consider if different forms of connectedness (e.g., family, peers, school)-previously found to have distinct relationship with suicidal thoughts and behavior among teens (Arango et al., 2018; Czyz,
Liu, \& King, 2012; Kaminski et al., 2010)—may be differentially associated with near-term risk.

Finally, the time-dependent relationship between suicidal thoughts and individual risk factors examined in this study points to the possibility that the dynamic nature of these constructs may require even more fine-grained assessment in order to improve short-term prediction. Identifying SI over short time intervals might also be improved by considering additional features of predictors (e.g. change scores), evaluating different contextual factors or mood states as well as the impact of treatment (attendance, adherence, type), or, as found in this study, by examining synergistic effects of risk indicators. A focus on distinct phenotypes of SI, accounting for its intensity and variability, in real-time (Kleiman et al., 2018) could offer another promising approach to short-term risk identification. Research employing frequent assessments across different populations as well as settings is needed to help address these research questions and ultimately help identify meaningful targets of intervention for individuals at elevated suicide risk.

\section{Acknowledgements}

Support for this project was provided by the American Foundation of Suicide Prevention (grant PDF-0-028-14) and by the Michigan Institute for Clinical and Health Research's Postdoctoral Translational Scholar Program. The authors thank Katherine Foster, M.S. for risk management support. The authors also gratefully acknowledge the clinical and administrative staff in the Child and Adolescent Psychiatric Inpatient Program at the University of Michigan, with a special thank you to Bernard Biermann, M.D., Ph.D. and Nicole Figueroa, M.S.N., R.N. for their invaluable support. The authors are grateful for the support of research staff of the University of Michigan's Youth Depression and Suicide Prevention Program and would also like to thank the youth and families who participated in this study. The authors have declared that they have no competing or potential conflicts of interest.

\section{Correspondence}

Ewa K. Czyz, Department of Psychiatry, University of Michigan, 4250 Plymouth Rd. Ann Arbor, MI 48109, USA.Email: ewac@umich.edu

\section{Key points}

- Little is known about the dynamic nature of suicidal ideation and its near-term association with risk factors; this study of adolescent utilized daily electronic diaries for a month after psychiatric hospitalization.

- Suicidal ideation, and its risk factors (hopelessness, connectedness, burdensomeness), fluctuated considerably day-to-day in the postdischarge period.

- These risk factors were concurrently, but not short-term prospectively, associated with suicidal ideation. Low connectedness combined with either high hopelessness or high burdensomeness was reliability associated with more severe same- and next-day ideation. 
- Fostering adolescents' sense of connectedness to others may be a promising intervention target to reduce thoughts of suicide in the high-risk postdischarge period.

- Capturing dynamic changes in suicidal ideation could improve near-term prediction of risk and identify potential intervention targets.

\section{Note}

${ }^{1}$ As reported elsewhere (Czyz, King, \& Biermann, 2018), adolescents in the intervention group reported more days (or events) they had thoughts of suicide across the 28-day follow-up; however, there were no group differences in terms of intensity of these thoughts (ideation frequency, duration, and urge severity). Additionally, there were no group differences based on predictors of interest: hopelessness, connectedness, and burdensomeness.

\section{References}

Arango, A., Cole-Lewis, Y., Lindsay, R., Yeguez, C.E., Clark, M., \& King, C. (2018). The protective role of connectedness on depression and suicidal ideation among bully victimized youth. Journal of Clinical Child \& Adolescent Psychology, in press.

Bagge, C.L., Glenn, C.R., \& Lee, H.J. (2013). Quantifying the impact of recent negative life events on suicide attempts. Journal of Abnormal Psychology, 122, 359-368.

Beck, A.T., Steer, R.A., Kovacs, M., \& Garrison, B. (1985). Hopelessness and eventual suicide: A 10-year prospective study of patients hospitalized with suicidal ideation. American Journal of Psychiatry, 1, 559-563.

Benjamini, Y., \& Hochberg, Y. (1995). Controlling the false discovery rate: A practical and powerful approach to multiple testing. Journal of the Royal Statistical Society, Series B, 57, 289-300.

Ben-Zeev, D., Young, M.A., \& Depp, C.A. (2012). Real-time predictors of suicidal ideation: Mobile assessment of hospitalized depressed patients. Psychiatry Research, 197, 55-59.

Bolland, J., McCallum, D., Lian, B., Bailey, C., \& Rowan, P. (2001). Hopelessness and violence among inner-city youths. Maternal and Child Health Journal, 5, 237-244.

Brent, D.A., McMakin, D.L., Kennard, B.D., Goldstein, T.R., Mayes, T.L., \& Douaihy, A.B. (2013). Protecting adolescents from self-harm: A critical review of intervention studies. Journal of the American Academy of Child \& Adolescent Psychiatry, 52, 1260-1271.

Bridge, J.A., Goldstein, T.R., \& Brent, D.A. (2006). Adolescent suicide and suicidal behavior. Journal of Child Psychology and Psychiatry, 47, 372-394.

Centers for Disease Control and Prevention. (2018). Web-based injury statistics query and reporting system (WISQARS). Available from: https://www.cdc.gov/injury/wisqars/inde x.html [last accessed 22 June 2018].

Chu, C., Buchman-Schmitt, J.M., Stanley, I.H., Hom, M.A., Tucker, R.P., Hagan, C.R., .. \& \& Michaels, M.S. (2017). The interpersonal theory of suicide: A systematic review and metaanalysis of a decade of cross-national research. Psychological Bulletin, 143, 1313-1345.

Chung, D.T., Ryan, C.J., Hadzi-Pavlovic, D., Singh, S.P., Stanton, C., \& Large, M.M. (2017). Suicide rates after discharge from psychiatric facilities: A systematic review and meta-analysis. JAMA Psychiatry, 74, 694-702.

Czyz, E.K., Berona, J., \& King, C.A. (2016). Rehospitalization of suicidal adolescents in relation to course of suicidal ideation and future suicide attempts. Psychiatric Services, 67, 332338 .
Czyz, E.K., \& King, C.A. (2015). Longitudinal trajectories of suicidal ideation and subsequent suicide attempts among adolescent inpatients. Journal of Clinical Child \& Adolescent Psychology, 44, 181-193.

Czyz, E.K., King, C.A., \& Biermann, B.J. (2018). Motivational Interviewing-enhanced safety planning for adolescents at high suicide risk: A pilot randomized controlled trial. Journal of Clinical Child \& Adolescent Psychology, in press.

Czyz, E.K., King, C.A., \& Nahum-Shani, I. (2018). Ecological assessment of daily suicidal thoughts and attempts among suicidal teens after psychiatric hospitalization: Lessons about feasibility and acceptability. Psychiatry Research, 267, 566-574.

Czyz, E.K., Liu, Z., \& King, C.A. (2012). Social connectedness and one-year trajectories among suicidal adolescents following psychiatric hospitalization. Journal of Clinical Child \& Adolescent Psychology, 41, 214-226.

Davidson, C.L., Anestis, M.D., \& Gutierrez, P.M. (2017). Ecological momentary assessment is a neglected methodology in suicidology. Archives of Suicide Research, 21, 111.

Fernandez, K.C., Johnson, M.R., \& Rodebaugh, T.L. (2013). TelEMA: A low-cost and user-friendly telephone assessment platform. Behavioral Research Methods, 45, 1279-1291.

Franklin, J.C., Ribeiro, J.D., Fox, K.R., Bentley, K.H., Kleiman, E.M., Huang, X., .. \& \& Nock, M.K. (2017). Risk factors for suicidal thoughts and behaviors: A meta-analysis of 50 years of research. Psychological Bulletin, 143, 187-232.

Gipson, P.Y., Agarwala, P., Opperman, K.J., Horwitz, A., \& King, C.A. (2015). Columbia-Suicide Severity Rating Scale: Predictive validity with adolescent psychiatric emergency patients. Pediatric Emergency Care, 31, 88-94.

Glenn, C.R., \& Nock, M.K. (2014). Improving the short-term prediction of suicidal behavior. American Journal of Preventive Medicine, 47, S176-S180.

Goldston, D.B., Daniel, S.S., Reboussin, D.M., Reboussin, B.A., Frazier, P.H., \& Kelley, A.E. (1999). Suicide attempts among formerly hospitalized adolescents: A prospective naturalistic study of risk during the first 5 years after discharge. Journal of the American Academy of Child \& Adolescent Psychiatry, 38, 660-671.

Hallensleben, N., Spangenberg, L., Forkmann, T., Rath, D., Hegerl, U., Kersting, A., .. \& \& Glaesmer, H. (2018). Investigating the dynamics of suicidal ideation. Crisis, 39, 65-69.

Hill, R.M., Rey, Y., Marin, C.E., Sharp, C., Green, K.L., \& Pettit, J.W. (2015). Evaluating the Interpersonal Needs Questionnaire: Comparison of the reliability, factor structure, and predictive validity across five versions. Suicide and LifeThreatening Behavior, 45, 302-314.

Horwitz, A.G., Czyz, E.K., \& King, C.A. (2015). Predicting future suicide attempts among adolescent and emerging adult psychiatric emergency patients. Journal of Clinical Child \& Adolescent Psychology, 44, 751-761.

Husky, M., Olié, E., Guillaume, S., Genty, C., Swendsen, J., \& Courtet, P. (2014). Feasibility and validity of ecological momentary assessment in the investigation of suicide risk. Psychiatry Research, 220, 564-570.

Husky, M., Swendsen, J., Ionita, A., Jaussent, I., Genty, C., \& Courtet, P. (2017). Predictors of daily life suicidal ideation in adults recently discharged after a serious suicide attempt: A pilot study. Psychiatry Research, 256, 79-84.

Joiner, T. (2005). Why people die by suicide. Cambridge, MA: Harvard University Press. 
Kaminski, J.W., Puddy, R.W., Hall, D.M., Cashman, S.Y., Crosby, A.E., \& Ortega, L.A.G. (2010). The relative influence of different domains of social connectedness on self-directed violence in adolescence. Journal of Youth \& Adolescence, 39, 460-473.

Kann, L., McManus, T., Harris, W.A., Shanklin, S.L., Flint, K.H., Queen, B., ... \& Ethier, K.A. (2018). Youth risk behavior surveillance - United States, 2017. MMWR Surveillance Summaries, 67, 1-114.

Kazdin, A.E., Rodgers, A., \& Colbus, D. (1986). The Hopelessness Scale for Children: Psychometric characteristics and concurrent validity. Journal of Consulting and Clinical Psychology, 54, 241-245.

Kleiman, E.M., Turner, B.J., Fedor, S., Beale, E.E., Huffman, J.C., \& Nock, M.K. (2017). Examination of real-time fluctuations in suicidal ideation and its risk factors: Results from two ecological momentary assessment studies. Journal of Abnormal Psychology, 126, 726-738.

Kleiman, E.M., Turner, B.J., Fedor, S., Beale, E.E., Picard, R.W., Huffman, J.C., \& Nock, M.K. (2018). Digital phenotyping of suicidal thoughts. Depression \& Anxiety, 35, 601608.

Law, M.K., Furr, R.M., Arnold, E.M., Mneimne, M., Jaquett, C., \& Fleeson, W. (2015). Does assessing suicidality frequently and repeatedly cause harm? A randomized control study. Psychological Assessment, 27, 1171-1181.

Mazza, J.J., \& Reynolds, W.M. (1998). A longitudinal investigation of depression, hopelessness, social support, and major and minor life events and their relation to suicidal ideation in adolescents. Suicide and Life-Threatening Behavior, 28, 358-374.

McLoughlin, A.B., Gould, M.S., \& Malone, K.M. (2015). Global trends in teenage suicide: 2003-2014. QJM: An International Journal of Medicine, 108, 765-780.

Moskowitz, D.S., \& Young, S.N. (2006). Ecological momentary assessment: What it is and why it is a method of the future in clinical psychopharmacology. Journal of Psychiatry and Neuroscience, 31, 13-20.

Nahum-Shani, I., Hekler, E.B., \& Spruijt-Metz, D. (2015). Building health behavior models to guide the development of just-in-time adaptive interventions: A pragmatic framework. Health Psychology, 34(S), 1209-1219.

Nahum-Shani, I., Smith, S.N., Spring, B.J., Collins, L.M., Witkiewitz, K., Tewari, A., \& Murphy, S.A. (2017). Just-in-time adaptive interventions (JITAIs) in mobile health: Key components and design principles for ongoing health behavior support. Annals of Behavioral Medicine, 52, 446-462.

Nock, M.K., Prinstein, M.J., \& Sterba, S.K. (2009). Revealing the form and function of self-injurious thoughts and behaviors: A real-time ecological assessment study among adolescents and young adults. Journal of Abnormal Psychology, 118, 816-827.

Opperman, K., Czyz, E.K., Gipson, P.Y., \& King, C.A. (2015). Connectedness and perceived burdensomeness among adolescents at elevated suicide risk: An examination of the interpersonal theory of suicidal behavior. Archives of Suicide Research, 19, 385-400.

Posner, K., Brown, G.K., Stanley, B., Brent, D.A., Yershova, K.V., Oquendo, M.A., ... \& Shen, S. (2011). The Columbia-
Suicide severity rating scale: Initial validity and internal consistency findings from three multisite studies with adolescents and adults. American Journal of Psychiatry, 168, 1266-1277.

Prinstein, M.J., Nock, M.K., Simon, V., Aikins, J.W., Cheah, C.S., \& Spirito, A. (2008). Longitudinal trajectories and predictors of adolescent suicidal ideation and attempts following inpatient hospitalization. Journal of Consulting and Clinical Psychology, 76, 92-103.

Ribeiro, J.D., Franklin, J.C., Fox, K.R., Bentley, K.H., Kleiman, E.M., Chang, B.P., \& Nock, M.K. (2016). Self-injurious thoughts and behaviors as risk factors for future suicide ideation, attempts, and death: A meta-analysis of longitudinal studies. Psychological Medicine, 46, 225-236.

Rudd, M.D., Berman, A.L., Joiner, T.E., Nock, M.K., Silverman, M.M., Mandrusiak, M., ... \& Witte, T. (2006). Warning signs for suicide: Theory, research, and clinical applications. Suicide and Life-Threatening Behavior, 36, 255-262.

Shiffman, S., Stone, A.A., \& Hufford, M.R. (2008). Ecological momentary assessment. Annual Review of Clinical Psychology, 4, 1-32.

Smith, J.M., Alloy, L.B., \& Abramson, L.Y. (2006). Cognitive vulnerability to depression, rumination, hopelessness, and suicidal ideation: Multiple pathways to self-injurious thinking. Suicide and Life-Threatening Behavior, 36, 443-454.

Spirito, A., \& Esposito-Smythers, C. (2006). Attempted and completed suicide in adolescence. Annual Review of Clinical Psychology, 2, 237-266.

Steer, R., Kumar, G., \& Beck, A. (1993). Self-reported suicidal ideation in adolescent psychiatric inpatients. Journal of Consulting and Clinical Psychology, 61, 1096-1099.

Stewart, S.M., Eaddy, M., Horton, S.E., Hughes, J., \& Kennard, B. (2017). The validity of the interpersonal theory of suicide in adolescence: A review. Journal of Clinical Child \& Adolescent Psychology, 46, 437-449.

Torous, J., Staples, P., Shanahan, M., Lin, C., Peck, P., Keshavan, M., \& Onnela, J.P. (2015). Utilizing a personal smartphone custom app to assess the patient health questionnaire-9 (PHQ-9) depressive symptoms in patients with major depressive disorder. JMIR Mental Health, 2, e8.

Van Orden, K.A., Cukrowicz, K.C., Witte, T.K., \& Joiner, T.E., Jr (2012). Thwarted belongingness and perceived burdensomeness: Construct validity and psychometric properties of the Interpersonal Needs Questionnaire. Psychological Assessment, 24, 197-215.

Van Orden, K.A., Witte, T.K., Cukrowicz, K.C., Braithwaite, S.R., Selby, E.A., \& Joiner, T.E., Jr (2010). The interpersonal theory of suicide. Psychological Review, 117, 575-600.

Wenzel, A., \& Beck, A.T. (2008). A cognitive model of suicidal behavior: Theory and treatment. Applied and Preventive Psychology, 12, 189-201.

Yen, S., Weinstock, L.M., Andover, M.S., Sheets, E.S., Selby, E.A., \& Spirito, A. (2013). Prospective predictors of adolescent suicidality: 6-month post-hospitalization follow-up. Psychological Medicine, 43, 983-993.

Accepted for publication: 2 August 2018

First published online: 24 September 2018 